Wissenschaftsgeschichte der Germanistik im 19. Jahrhundert 


\title{
Wissenschaftsgeschichte der Germanistik im 19. Jahrhundert
}

\author{
Herausgegeben von \\ Jürgen Fohrmann und Wilhelm Voßkamp
}

Mit Beiträgen von Uwe Meves, Rainer Kolk, Herbert H. Egglmaier, Ulrich Hunger, Rüdiger Krohn, Nikolaus Wegmann, Hans-Martin Kruckis, Holger Dainat, Cornelia Fiedeldey-Martyn, Jürgen Fohrmann, Maximilian Nutz, Christian Grawe, Detlev Kopp 
Die Deutsche Bibliothek - CIP-Einheitsaufnahme

Wissenschaftsgeschichte der Germanistik im 19. Jahrhundert / hrsg. von Jürgen Fohrmann und Wilhelm Voßkamp. Mit Beitr. von Uwe Meves ...- Stuttgart ; Weimar : Metzler, 1994 ISBN 978-3-476-00990-6

NE: Fohrmann, Jürgen [Hrsg.]; Meves, Uwe

ISBN 978-3-476-00990-6

ISBN 978-3-476-03523-3 (eBook)

DOI $10.1007 / 978-3-476-03523-3$

Dieses Werk einschließlich aller seiner Teile ist urheberrechtlich geschützt.

Jede Verwertung außerhalb der engen Grenzen des Urheberrechtsgesetzes ist ohne Zustimmung des Verlages unzulässig und strafbar. Das gilt insbesondere für Vervielfältigungen, Übersetzungen, Mikroverfilmungen und die Einspeicherung und Verarbeitung in elektronischen Systemen.

(C) 1994 Springer-Verlag GmbH Deutschland

Ursprünglich erschienen bei J.B. Metzlersche Verlagsbuchhandlung und Carl Ernst Poeschel Verlag GmbH in Stuttgart 1994 


\section{Vorwort}

Die vorliegende Darstellung zur Geschichte der Germanistik ist weitgehend aus dem von der Deutschen Forschungsgemeinschaft über mehrere Jahre unterstützten Projekt zur »Geschichte der deutschen Literaturwissenschaft im 19. Jahrhundert« hervorgegangen. Für die großzügige Förderung, die auch noch einen Zuschuß für die Drucklegung einschloß, sind die Herausgeber der DFG zu großem Dank verpflichtet. Daneben möchten wir auch jenen Kollegen danken, die die Projektarbeit begleiteten und am Ende bereit waren, ihre Beiträge für den Band zur Verfügung zu stellen.

Unser Dank gilt ebenfalls den Bielefelder und Kölner Universitätsbibliotheken, ohne deren kooperatives Verhalten eine solche umfassende Forschungsarbeit nicht möglich gewesen wäre.

Großer Dank ist schließlich Gerd Müller und Hedwig Pompe abzustatten, die die Drucklegung der Publikation besorgten und Petra Trösch, die das Personenregister erstellte.

Der J.B. Metzlerschen Verlagsbuchhandlung sind wir für die Aufnahme in ihr Verlagsprogramm verbunden.

Der nun vorliegende Band gibt in seinen bibliographischen Teilen den Stand der wissenschaftsgeschichtlichen Diskussion bis zum Jahre 1989 wieder. Für die nachfolgenden Jahre verweist er auf die "Marbacher Mitteilungen« des »Marbacher Arbeitskreises für die Geschichte der Germanistik«, die periodisch eine jeweils umfassende Bibliographie zum Thema anbieten.

Bonn und Köln, im Dezember 1993 Jürgen Fohrmann, Wilhelm Voßkamp 


\section{Inhalt}

\section{Einleitung}

JÜRGEN FOHRMANN

Von den deutschen Studien zur Literaturwissenschaft . . . . . . . . . . 1

WiLHELM VOSSKAMP

〉Bildung als Synthese $\ldots \ldots \ldots \ldots \ldots \ldots \ldots$

\section{Der Name}

UWE MEVES

Zur Namensgebung $>$ Germanistik $\ldots \ldots \ldots \ldots \ldots \ldots$

\section{Institutionengeschichte}

\section{RAINER KOLK}

Liebhaber, Gelehrte, Experten. Das Sozialsystem der Germanistik bis zum Beginn des 20. Jahrhunderts . . . . . . . . . . . . Vorbemerkung 48 - Zur Erforschung deutscher Sprache und Literatur vor 180049 - Anfänge und institutionelle Etablierung der Germanistik (1800-1840) $55 \cdot$ Konsolidierung und Kontroverse 1840-1880 $79 \cdot \mathrm{Ak}$ tualisierungen und Neuansätze (1880-1910) 97

\section{Uwe Meves}

Zum Institutionalisierungsprozeß der Deutschen Philologie:

Die Periode der Lehrstuhlerrichtung . . . . . . . . . . . . . . . . . .

Vorbemerkung 115 • Ausgangspunkt: Die Situation um 1810118 - Etablierungsphase: Von 1810 bis zum Ende der 40er Jahre 129 · Das Fach Deutsche Philologie und die Lehrerausbildung 150 - Der Beginn der Konsolidierungsphase: Die 50er Jahre 165 • Das Fach Deutsche Philologie auf dem Weg zur Brotwissenschaft 177 • Der Abschluß der Konsolidierungsphase: Die 60er Jahre 186 ' Schlußbemerkung 194 ' Anhang: Tabellen 197 
Herbert H. EgGLMaIER

Entwicklungslinien der neueren deutschen Literaturwissenschaft in Österreich in der zweiten Hälfte des 19. Jahrhunderts und zu Beginn des 20. Jahrhunderts . . . . . . . . . . . . . . . . . . . . .

\section{Altgermanistik}

\section{ULRICH HUNGER}

Die altdeutsche Literatur und das Verlangen nach Wissenschaft.

Schöpfungsakt und Fortschrittsglaube in der Frühgermanistik . . . . .

\section{RÜDIGER KROHN}

»... daß Alles Allen verständlich sey...«. Die Altgermanistik

des 19. Jahrhunderts und ihre Wege in die Öffentlichkeit . . . . . . . . . .

Sicherung des Überlieferten 267 . Poetische Wiedergewinnung 276 .

Rückkehr zu den Originalen 286 . Kommentare als Verständnishilfen 292 · Mehrstufige Annäherung 301 · Selbst-Verständlichkeit des Textes 309 - Verständlichkeit als Programm 319

\section{Philologie, Neugermanistik, Selbstreflexion}

\section{Nikolaus Wegmann}

Was heißt einen `klassischen Text lesen? Philologische Selbstreflexion zwischen Wissenschaft und Bildung ..............

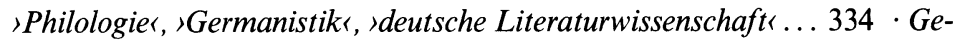
lehrte Philologie. Krise und Neuformierung 337 · Positiver Wert und didaktischer Zweck. Philologie als finale Einheit (Friedrich August Wolf) 353 - Philosophie der Philologie. Philologie als operative Einheit (Friedrich Schlegel und August Boeckh) 371 - Selbstreflexion als Klassiker-Philologie? Philologische Lektüre zwischen Moral und Epistemologie (Karl Lachmann) 399 • 'Wissenschaftı vs. >Bildungı. Selbstreflexion als disziplinäre Selbstkritik (Friedrich Nietzsche) 419 • Bibliographie 441

\section{HANS-MARTIN KRUCKIS}

Goethe-Philologie als Paradigma neuphilologischer Wissenschaft im 19. Jahrhundert . . . . . . . . . . . . . . . . . . . .

Frühe neuphilologische Programme 451 ' Empirismus und Erbauung: Danzels Programm einer kommenden Goethe-Philologie 455 - GoethePhilologie als nationale Bildung 463 - Institutionalisierungsschwierigkeiten und Autopoiesis der Mikrologie 472 . Scherer und das Ende der sklassischen، Goethe-Philologie 480

\section{Holger DaINAT}

Von der Neueren Deutschen Literaturgeschichte zur Literaturwissenschaft. Die Fachentwicklung von 1890 bis $1913 / 14$. . . . . . . . . 
Holger Dainat/CoRnelia Fiedeldey-Martyn

Literaturwissenschaftliche Selbstreflexion. Eine Bibliographie, $1792-1914 \ldots \ldots \ldots \ldots \ldots \ldots \ldots \ldots \ldots \ldots \ldots$

\section{Wissenschaftliche Genres}

\section{HANS-MARTIN KRUCKIS}

Biographie als literaturwissenschaftliche Darstellungsform

im 19. Jahrhundert . . . . . . . . . . . . . . . . . . . . . . . . .

JÜRGEN FOHRMANN

Geschichte der deutschen Literaturgeschichtsschreibung

zwischen Aufklärung und Kaiserreich . . . . . . . . . . . . . .

\section{Öffentlichkeit und Klassikerrezeption}

\section{MaXimilian NutZ}

Das Beispiel Goethe. Zur Konstituierung eines nationalen Klassikers . Zur Genese des Ruhms: Repräsentant und Projektionsfigur 609 • Arbeit am eigenen Denkmal 613 - Lehrmeister personaler Bildung 617 •Von der negativen Symbolfigur zum nationalen Klassiker 621 · Identitätsfindung und Besitzerstolz 624 - Institutionalisierung und Instrumentalisierung eines >Kults` 629 • Die Etablierung einer Wissenschaft als GoethePhilologie 633

\section{Christian Grawe}

Das Beispiel Schiller. Zur Konstituierung eines Klassikers in der Öffentlichkeit des 19. Jahrhunderts

\section{Literaturwissenschaft und Deutschunterricht}

\section{DETLEV KoPP}

(Deutsche) Philologie und Erziehungssystem . . . . . . . . . . . . .

Klassische Philologie, Lateinunterricht und Deutschunterricht bis 1800669 - Philologie(n) und gymnasialer Unterricht im 19. Jahrhundert 695

\section{Bibliographien}

\section{CoRnelia Fiedeldey-MARTYN}

Bibliographie zur Wissenschaftsgeschichte der deutschen Literatur-

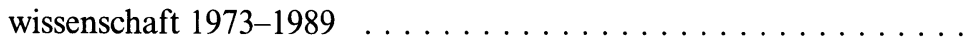

